\title{
A High Ductal Flow Velocity Is Associated with Successful Pharmacological Closure of Patent Ductus Arteriosus in Infants 22-27 Weeks Gestational Age
}

\author{
Karl Wilhelm Olsson, Anders Jonzon, and Richard Sindelar \\ Department of Women's and Children's Health, Uppsala University Children's Hospital, \\ Uppsala University, SE-751 85 Uppsala, Sweden \\ Correspondence should be addressed to Richard Sindelar, richard.sindelar@kbh.uu.se
}

Received 4 September 2012; Revised 10 November 2012; Accepted 10 November 2012

Academic Editor: Gustavo Rocha

Copyright (C 2012 Karl Wilhelm Olsson et al. This is an open access article distributed under the Creative Commons Attribution License, which permits unrestricted use, distribution, and reproduction in any medium, provided the original work is properly cited.

Objective. To identify factors affecting closure of patent ductus arteriosus (PDA) in newborn infants born at 22-27 weeks gestational age (GA) during pharmacological treatment with cyclooxygenase inhibitors. Method. Infants born at 22-27 weeks of GA between January 2006 and December 2009 who had been treated pharmacologically for PDA were identified retrospectively. Medical records were assessed for clinical, ventilatory, and outcome parameters. Echocardiographic examinations during treatment were reviewed. Results. Fifty-six infants were included in the study. Overall success rate of ductal closure with pharmacological treatment was $52 \%$. Infants whose PDA was successfully closed had a higher GA $(25+4$ weeks versus $24+3$ weeks; $P=0.047)$, and a higher pretreatment left to right maximal ductal flow velocity $(1.6 \mathrm{~m} / \mathrm{s}$ versus $1.1 \mathrm{~m} / \mathrm{s} ; P=0.023)$. Correcting for GA, preeclampsia, antenatal steroids, and age at start of treatment, a higher maximal ductal flow velocity was still associated with successful ductal closure (OR 3.04; $P=0.049$ ). Conclusion. Maximal ductal flow velocity was independently associated with success of PDA treatment.

\section{Introduction}

Infants born before 28 gestational weeks have a high incidence of patent ductus arteriosus (PDA) $[1,2]$. The postnatal presence of a haemodynamically significant left to right shunt through the duct is associated with a lower survival rate and an increased incidence of intraventricular haemorrhage (IVH), necrotizing enterocolitis (NEC), and bronchopulmonary dysplasia in preterm infants [3-9]. Inhibition of prostaglandin production with the cyclooxygenase inhibitors indomethacin or ibuprofen is the standard pharmacological treatment for PDA [10]. Nevertheless failure of pharmacologic treatment frequently occurs in extremely preterm infants and is associated with increased mortality [11-13].

Previous studies have identified low gestational age (GA), pregnancy-induced hypertension, antenatal indomethacin exposure, lack of antenatal glucocorticoid exposure, late indomethacin treatment, respiratory distress syndrome (RDS), use of high-frequency oscillatory ventilation (HFOV), large ductal diameter, and less ductal shunt velocity as independent risk factors for failure of pharmacological treatment of PDA [14-18].

Pulmonary factors such as prenatal steroid exposure and RDS thus appear to affect the closure of the ductus arteriosus, and parameters related to pulmonary circulation, for example, high $\mathrm{PaO}_{2}$ and low blood pressure within the ductus arteriosus, relate to physiological ductal constriction in animal studies [19]. The objective of this retrospective study was to identify factors associated with closure of the ductus arteriosus during treatment with cyclooxygenase inhibitors in infants born at 22-27 weeks GA, with special focus on ventilatory and pulmonary circulatory factors.

\section{Patients and Methods}

2.1. Patients. Infants born at Uppsala University Children's Hospital between January 2006 and December 2009 at a GA of less than 28 weeks and pharmacologically treated for PDA 
were included in this retrospective cohort study. Infants with any major congenital anomalies were excluded. The study was approved by the Swedish Central Ethical Review Board.

2.2. Pharmacological Treatment for Patent Ductus Arteriosus. All newborn infants born at a GA of less than 28 weeks were evaluated echocardiographically within the first days of life and echocardiographic examinations were repeated if indicated. Examinations were performed using an Acuson Sequoia Ultrasound System (Siemens AB, Upplands Väsby, Sweden) with a $10 \mathrm{MHz}$ transducer and results were saved in digital format (Xcelera, Philips AB, Stockholm, Sweden).

A haemodynamically significant PDA was defined by one or more of the following echocardiographic parameters: ductus arteriosus diameter of $>1.5 \mathrm{~mm}$; left atrium to aortic root ratio (LA/Ao) of $>1.5$; reduced and reversed flow during diastole in the descending aorta in combination with clinical signs. Pharmacological treatment was initiated when a PDA of haemodynamic significance was found and none of the following contraindications were present: ductal-dependent heart defect; renal failure (serum creatinine $>120 \mu \mathrm{mol} / \mathrm{L}$ or serum urea $>12 \mathrm{mmol} / \mathrm{L}$ ); thrombocytopenia (platelets $\left.<50 \times 10^{9} / \mathrm{L}\right)$; IVH grade II-IV; or NEC. Indomethacin (Indocid, Merck \& Co., Inc., West Point, Pennsylvania, USA) was administered in a three-dose regimen as an infusion $(0.2 \mathrm{mg} / \mathrm{mL}, 0.2 \mathrm{mg} / \mathrm{kg} /$ dose $)$ over at least 20 minutes per dose, the second dose 12 hours after the first, and the third dose 24 hours after the second. If echocardiography revealed a patent ductus after these three doses, one to three additional doses were administered at 24 hours intervals guided by echocardiographic examinations after each additional dose. No additional pharmacological ductus treatment was administered. Surgical ligation was carried out if signs of a hemodynamically significant ductus arteriosus persisted after pharmacological treatment. Before discharge a clinical assessment of ductus arteriosus was made and additional echocardiographic examination performed if indicated.

2.3. Concomitant Treatment. According to the policy at the unit, infants received mechanical ventilatory support immediately after birth and early surfactant (Curosurf, Nycomed International, Zürich, Switzerland) administration $(100 \mathrm{mg} / \mathrm{kg})$ if signs of respiratory insufficiency were detected. Otherwise early nasal continuous positive airway pressure (CPAP) therapy was instituted. HFOV was used as rescue treatment.

Guideline fluid volumes were $90-100 \mathrm{~mL} / \mathrm{kg}$ during the first day of life, adding $10 \mathrm{~mL} / \mathrm{kg} /$ day each day during the first week for infants with a GA of 24 weeks or less, and $80-90 \mathrm{~mL} / \mathrm{kg}$, adding $10 \mathrm{~mL} / \mathrm{kg} /$ day for infants with a GA of 25 to 27 weeks. Fluid intake was further individually adjusted, guided by weight loss, urinary output, and serum sodium concentration. Volume substitution and inotropic drugs were used restrictively and only when combinations of low mean arterial blood pressure and low micturition were detected. Bacterial cultures were taken and intravenous administration of antibiotics initiated if clinical suspicion of bacterial septicemia arose.
2.4. Perinatal Characteristics and Outcome at Discharge. Medical records were retrospectively assessed for information regarding GA, birth weight, gender, preeclampsia, antenatal steroids, Caesarean section, surfactant administration and Apgar-scores, and death. Nursing flow charts from the neonatal intensive care unit were assessed for details about PDA treatment, including information about ventilatory settings and fluid administration.

2.5. Echocardiography. Each infant's last echocardiographic examination before treatment start was reassessed for this study by a single cardiologist (A. Jonzon), who was blinded to treatment results. Maximal ductal flow velocity was assessed from the parasternal short axis view with pulsed and continuous Doppler directly in line with the ductal flow and ductal diameter was measured at the ductus narrowest inner dimension from the same position with and without color Doppler. Left atrium to aortic root ratio was measured in Mmode from the parasternal long axis.

The first follow-up echocardiography after treatment defined successful (Closed group) or failed (Persistent group) ductal closure. The PDA was considered closed if no ductal flow could be found with color Doppler.

2.6. Statistical Analysis. Data for each group is presented below as median values and range or number and percentage. Statistical analyses were conducted with SPSS Statistics 18 for Windows (SPSS, Inc., Chicago, Illinois, USA). The Mann-Whitney test was used to compare non-parametric continuous data and the Fisher's exact test was used to compare categorical data. All $P$ values presented are twotailed and a $P<0.05$ was considered statistically significant. Multivariable logistic regression was performed to assess the individual influence of predictive factors on the proportion of ductal closure. Factors previously found to affect ductal closure during treatment (GA, preeclampsia, antenatal glucocorticoid administration, time of treatment start) were included in the analyses together with maximal ductal shunt velocity adjusted for the second power of ductal diameter and the time of echocardiography. The adjustment for second power of ductal diameter was made to assess whether flow velocity was independent of ductal diameter according to the Hagen-Poiseuille equation.

\section{Results}

Between January 2006 and December 2009, a total of 130 infants were born at 22-27 weeks of GA at Uppsala University Children's Hospital. Fifty-six infants received pharmacological treatment for patency of the ductus arteriosus and 6 infants received primary surgical treatment because of contraindications for pharmacological treatment before discharge from the same neonatal unit. In 18 infants the PDA closed spontaneously without treatment, 13 died and 37 did not receive pharmacological treatment before discharge because of either contraindications for treatment or a PDA not considered haemodynamically significant. Out of the 56 pharmacologically treated infants, 29 (52\%) showed 
Table 1: Perinatal characteristics.

\begin{tabular}{lccc}
\hline & Ductus closed $(n=29)$ & Ductus persistent $(n=27)$ & $P$ \\
\hline Gestational age, weeks (range) & $25^{+5}\left(22^{+2}-27^{+4}\right)$ & $24^{+3}\left(22^{+3}-27^{+4}\right)$ & $\mathbf{0 . 0 4 7}$ \\
Birth weight, grams (range) & $718(432-1217)$ & $595(440-1052)$ & 0.363 \\
Male gender (\%) & $20(69)$ & $14(52)$ & 0.274 \\
Preeclampsia (\%) & $5(17)$ & $22(81)$ & 1.000 \\
Antenatal steroids (\%) & $23(79)$ & $15(56)$ & 1.000 \\
Cesarean section (\%) & $17(59)$ & $26(96)$ & 1.000 \\
Surfactant (\%) & $28(97)$ & & 1.000 \\
\hline
\end{tabular}

successful PDA closure (Closed group) and 27 (48\%) failed to close (Persistent group).

3.1. Perinatal Characteristics. All observed perinatal characteristics were similar in the two groups with the exception of GA, which was higher in the Closed group (Table 1). No infant had been exposed to antenatal indomethacin. Median Apgar-scores at one, five, and ten minutes were 5, 7, and 9 in the Closed group and 5, 8, and 9 in the Persistent group $(P=0.980,0.807$, and 0.773 , resp.).

3.2. Echocardiography. All infants had a predominately left to right ductal flow. Besides a higher maximal ductal flow velocity in the Closed group compared to the Persistent group, no other differences in echocardiographic parameters or ventilator characteristics were observed at the time of the last echocardiographic examination before treatment (Table 2).

\subsection{Pharmacological PDA and Concomitant Treatment Char-} acteristics. No major differences in treatment characteristics or fluid intake during treatment were observed between the two groups studied (Table 3 ). All studied infants treated for PDA had been given indomethacin except eight, who received treatment with ibuprofen alone (Pedea, Orphan Europe SARL, Paris La Défense, France, $5 \mathrm{mg} / \mathrm{mL}$, first dose $10 \mathrm{mg} / \mathrm{kg} / \mathrm{dose}$ and $5 \mathrm{mg} / \mathrm{kg} /$ dose 24 and 48 hours after first dose) and two infants who received both indomethacin and ibuprofen due to shortage of indomethacin during part of the studied period. Two infants in each group received only two doses of indomethacin $(P=1.000)$ because of contraindications for treatment. In three infants in the Persistent group, treatment had been initiated late after birth at 20,24, and 40 days, respectively.

Multivariate logistical regression analysis for factors previously found to affect ductal closure during pharmacological PDA closure and maximal ductal shunt flow velocity (adjusted for squared ductal diameter and for time of echocardiography) still showed an association between higher maximal ductal flow velocity and ductal closure (Table 4).

3.4. Outcome at Discharge. Three infants in the Closed group had not undergone post-treatment echocardiography before discharge, but were considered clinically closed. They were discharged in good condition and have not been subjected to any further examination or treatment for ductus arteriosus since then. In the Persistent group, eleven infants (41\%) were subjected to surgery after follow up echocardiography, one infant $(4 \%)$ received a second course of ibuprofen at a regional hospital, three infants $(11 \%)$ died with an open ductus, five infants (19\%) had spontaneous closure of their PDA at the time of echocardiographic examination before discharge, and seven infants $(26 \%)$ were discharged with an open ductus. Three infants (10\%) in the Closed group had reopened PDAs since the first echocardiographic examination after treatment when repeatedly examined at 10,23 , and 28 days after treatment, respectively.

Three infants (10\%) in the Closed group and $4(15 \%)$ in the Persistent group died $(P=0.700)$ at a median of 38 (range 22-42) and 17 (range 6-166) days $(P=0.480)$, but no death was related to patency of the ductus arteriosus.

Using the Swedish Perinatal Quality Register (a national register for quality control of neonatal care) and nursing flow charts from the neonatal intensive care unit, information regarding use of HFOV was collected. Medical records were retrospectively assessed for information regarding diagnosis of culture-proven episodes of sepsis in connection to PDA treatment, bronchopulmonary dysplasia (BPD, defined as a need for supplemental oxygen at 36 weeks GA), periventricular leukomalacia (PVL), IVH (including grade), retinopathy of prematurity (ROP), and NEC.

Eleven infants in the Closed group and 17 infants in the Persistent group were on ventilator treatment at the start of PDA treatment $(P=0.108)$. Infants in the Closed group received CPAP therapy for a median of 46 (range 3-95) days and were on ventilator treatment for a median of 8 (range 0-65 days) whereas infants in the Persistent group received CPAP therapy for a median of 42 (range $0-122$ ) days and were on ventilator treatment for a median of 23 (range 0 100 ) days $(P=0.533$ and 0.100 , respectively). Four infants $(14 \%)$ in the Closed group and seven infants $(26 \%)$ in the Persistent group had undergone HFOV $(P=0.322)$, with a median of nine days (range 7-30) in the Closed group and four days (range 1-11) in the Persistent group $(P=$ 0.037). None of the infants in the Closed group and two (7\%) infants in the Persistent group had undergone HFOV before or during pharmacological treatment for PDA $(P=$ 0.228). None of the infants in the Closed group and two (7\%) infants in the Persistent group had undergone HFOV before or during pharmacological treatment for PDA $(P=$ $0.228)$. One $(3 \%)$ versus two (7sepsis in connection to PDA treatment $(P=0.605)$.

Twenty-nine $(100 \%)$ versus $27(100 \%)$ infants were diagnosed with RDS $(P=1.000), 15(52 \%)$ versus $16(59 \%)$ 
TABLE 2: Characteristics at time of echocardiography.

\begin{tabular}{|c|c|c|c|}
\hline & Ductus closed $(n=29)$ & Ductus persistent $(n=27)$ & $P$ \\
\hline Age at echocardiography, days (range) & $2(0-7)$ & $2(0-33)$ & 0.079 \\
\hline Ventilator $(\%)$ & $10(34)$ & $16(59)$ & 0.108 \\
\hline Ventilator $\mathrm{MAP}, \mathrm{cmH}_{2} \mathrm{O}$ (range) & $8(6-14)$ & $9(7-12)$ & 0.220 \\
\hline CPAP $(\%)$ & $19(66)$ & $11(41)$ & 0.108 \\
\hline $\mathrm{CPAP}$ pressure, $\mathrm{cmH}_{2} \mathrm{O}$ (range) & $5(4-7)$ & $5(3-7)$ & 0.618 \\
\hline Fraction of inspired oxygen, \% (range) & $25(21-52)$ & $27(21-42)$ & 0.848 \\
\hline Systolic blood pressure ${ }^{1}, \mathrm{mmHg}$ (range) & $47(37-62)$ & $47(35-84)$ & 0.987 \\
\hline Ductal diameter, mm (range) & $1.7(0.9-3.0)$ & $1.8(1.0-3.0)$ & 0.399 \\
\hline Maximal ductal flow velocity, m/s (range) & $1.6(0.5-2.7)$ & $1.1(0.7-2.9)$ & 0.023 \\
\hline LA/Ao (range) & $1.5(1.2-2.8)$ & $1.7(1.1-3.5)$ & 0.198 \\
\hline
\end{tabular}

${ }^{1} 21$ versus 15 infants had an arterial catheter which enabled blood pressure measurements.

TABle 3: Treatment characteristics.

\begin{tabular}{lccc}
\hline & Ductus closed $(n=29)$ & Ductus persistent $(n=27)$ & $P(1-40)$ \\
\hline Age at treatment start, days (range) & $3(1-8)$ & $22(82)$ & 0.117 \\
Indomethacin $(\%)$ & $26(90)$ & $6(22)$ & 0.462 \\
Ibuprofen $^{1}(\%)$ & $4(14)$ & $4(-12-12)$ & 0.497 \\
Change in weight, \% (range) & $1(-10-9)$ & $139(111-203)$ & 0.090 \\
Fluid intake, $\mathrm{mL} / \mathrm{kg} /$ day (range) & $134(98-168)$ & $43(0-89)$ & 0.354 \\
Part IV, \% (range) & $59(0-85)$ & $2.2(0.4-4.9)$ & 0.468 \\
Urine output, $\mathrm{mL} / \mathrm{kg} / \mathrm{h}$ (range) & $2.1(0.5-4.2)$ & 0.594 \\
\hline
\end{tabular}

${ }^{1} 1$ versus 1 infants received treatment with both indomethacin and ibuprofen during the same course.

TABle 4: Multivariable analysis for ductal closure.

\begin{tabular}{lcc}
\hline & OR (95\% CI) & $P$ \\
\hline Gestational age $^{1}$ & $1.45(0.93-2.25)$ & 0.103 \\
Preeclampsia & $0.78(0.11-5.60)$ & 0.807 \\
Antenatal steroids & $0.83(0.17-4.04)$ & 0.817 \\
Age at treatment start ${ }^{2}$, days & $0.82(0.60-1.12)$ & 0.213 \\
Maximal ductal flow velocity ${ }^{3}, \mathrm{~m} / \mathrm{s}$ & $3.04(1.01-9.22)$ & $\mathbf{0 . 0 4 9}$ \\
\hline${ }^{1}$ OR for every 1 week increase. & & \\
${ }^{2}$ OR for every 1 day increase. & & \\
${ }^{3}$ OR for every 1 m/s increase, adjusted for age at echocardiography and \\
squared ductal diameter.
\end{tabular}

with BPD $(P=0.602), 3(10 \%)$ versus $4(15 \%)$ with PVL $(P$ $=0.700), 5(17 \%)$ versus $7(26 \%)$ with IVH $(P=0.523), 15$ $(52 \%)$ versus $15(56 \%)$ with $\operatorname{ROP}(P=0.795)$ and $1(3 \%)$ versus $4(15 \%)$ with NEC $(P=0.185)$ in the Closed and Persistent groups, respectively. One infant in each group had IVH grade III-IV $(P=1.000)$, and all others were grade I-II.

\section{Discussion}

Our study shows that higher gestational age and maximal shunt velocity is associated with successful pharmacological PDA treatment in infants born at 22-27 weeks GA. No other factor was found to differ between infants whose PDA closed and infants whose PDA did not close during treatment. In a multivariable logistic regression analysis, including the factors GA, preeclampsia, prenatal steroids, age at treatment start, and maximal ductal flow velocity adjusted for ductal diameter, only maximal ductal flow velocity was found to be independently associated with ductal closure. Furthermore, our study could not confirm any significant difference in outcome between infants whose PDA did or did not close during treatment.

Although the effect of ductal flow velocity on ductal closure has previously been noted in a more mature cohort of newborn infants, the mechanisms behind it are not extensively studied $[14,15,20]$. Assuming the ductus arteriosus to resemble a cylindrical pipe, the Hagen-Poiseuille equation states that the flow velocity in the ductus arteriosus is proportional to the pressure gradient between the systemic to pulmonary circulation and to the second power of the ductal diameter while it is inversely proportional to the blood viscosity and the ductal length [21]. In one previous study, the difference in maximal ductal flow velocity between infants whose ductus did or did not close has been suggested to be attributed to a difference in pulmonary arterial pressure [14]. In our study the influence of maximal ductal flow velocity was independent of the second power of the ductal diameter. Due to the retrospective design of our study we did not have the possibility to measure and adjust for blood viscosity or ductal length and we could only obtain data on the systolic blood pressure measured by arterial catheter at the time of echocardiography from 21 infants in the Closed group and 15 infants in the Persistent group. The uniformity in the measured systolic blood pressures between the two groups suggests however that a difference in the systemic 
to pulmonary circulation pressure gradient likely reflects a higher pulmonary arterial pressure in the Persistent group.

Pulmonary vascular resistance normally decreases rapidly with the start of ventilation and oxygenation after birth, reversing the fetal right to left flow through the ductus arteriosus and foramen ovale [22]. The normal physiological ductus arteriosus closure occurs in two stages, where the initial contraction of the vessel is a response to the decrease in pulmonary vascular resistance and pressure, an increase in arterial oxygen pressure, a decrease in circulating prostaglandin E2 (PGE2), and a decrease in PGE2-receptors in the ductal wall $[19,23-25]$. Besides the vascular effect of a higher pulmonary pressure on the ductus arteriosus, the resulting lower blood flow and lower oxygen exposure of the ductus would therefore prevent an effective closing with pharmacological treatment. The previous finding of RDS as a risk factor for failure of pharmacological treatment of PDA underlines the close connection between ventilation, pulmonary circulation and the existence of a PDA $[16,18]$. The lack of any major difference in ventilatory parameters and $\mathrm{FiO}_{2}$ between the Closed and Persistent groups in our study could indicate that ductal flow may be more sensitive to assess pulmonary vascular resistance at this early stage of life in extremely preterm infants.

Our study is limited by its retrospective design, the exclusion of a number of infants with PDA that were not treated before discharge, and the use of both indomethacin and ibuprofen during the study period. However, the two groups were well balanced and the study had the advantage of reflecting clinical treatment decisions based on strict guidelines.

\section{Conclusion}

In summary this study indicates that the maximal ductal flow velocity, independently of ductal diameter, is associated with successful treatment of PDA in extremely preterm infants. Pre-treatment echocardiographic maximal ductal flow velocity could possibly be used to assess the chances for treatment success in individual infants, but more information on the reliability of this parameter is needed.

\section{Conflict of Interests}

The authors have indicated that they have no conflict of interests relevant to this paper to disclose.

\section{Acknowledgments}

This study was supported by Grants from Her Royal Highness the Crown Princess Lovisa's Foundation for Children's Health Care, Stockholm, and the Gillbergska Foundation, Uppsala. The sponsors had no involvement in the study design, the collection, analysis, or interpretation of data, the writing of the report, or the decision to submit the paper for publication. The authors would like to thank secretary Elisabeth Sigurdsson, NICU, Uppsala University Children's Hospital, Uppsala for her assistance in collection of clinical data and statistician Lars Berglund, Uppsala Clinical
Research Center, for his assistance with statistics. The authors would also like to thank the Swedish Heart-Lung Foundation and the Swedish Children's Heart Foundation for economic support enabling the poster presentation of the preliminary results of this study at the Pediatric Academic Societies Annual Meeting in Vancouver, Canada, May 1-4, 2010.

\section{References}

[1] K. Costeloe, E. Hennessy, A. T. Gibson, N. Marlow, and A. R. Wilkinson, "The EPICure study: outcomes to discharge from hospital for infants born at the threshold of viability," Pediatrics, vol. 106, no. $4 \mathrm{I}$, pp. 659-671, 2000.

[2] J. Wyllie, "Treatment of patent ductus arteriosus," Seminars in Neonatology, vol. 8, no. 6, pp. 425-432, 2003.

[3] G. G. Dudell and W. M. Gersony, "Patent ductus arteriosus in neonates with severe respiratory disease," Journal of Pediatrics, vol. 104, no. 6, pp. 915-920, 1984.

[4] J. M. Perlman, A. Hill, and J. J. Volpe, "The effect of patent ductus arteriosus on flow velocity in the anterior cerebral arteries: ductal steal in the premature newborn infant," Journal of Pediatrics, vol. 99, no. 5, pp. 767-771, 1981.

[5] F. D. Dykes, A. Lazzara, and P. Ahmann, "Intraventricular hemorrhage: a prospective evaluation of etiopathogenesis," Pediatrics, vol. 66, no. 1, pp. 42-49, 1980.

[6] N. Evans and M. Kluckow, "Early ductal shunting and intraventricular haemorrhage in ventilated preterm infants," Archives of Disease in Childhood, vol. 75, no. 3, pp. F183-F186, 1996.

[7] R. W. Ryder, J. D. Shelton, and M. E. Guinan, "Necrotizing entercolitis. A prospective multicenter investigation," American Journal of Epidemiology, vol. 112, no. 1, pp. 113-123, 1980.

[8] S. Dollberg, A. Lusky, and B. Reichman, "Patent ductus arteriosus, indomethacin and necrotizing enterocolitis in very low birth weight infants: a population-based study," Journal of Pediatric Gastroenterology and Nutrition, vol. 40, no. 2, pp. 184-188, 2005.

[9] D. Hayes, D. J. Feola, B. S. Murphy, L. A. Shook, and H. O. Ballard, "Pathogenesis of bronchopulmonary dysplasia," Respiration, vol. 79, no. 5, pp. 425-436, 2010.

[10] C. Hammerman, "Patent ductus arteriosus: clinical relevance of prostaglandins and prostaglandin inhibitors in PDA pathophysiology and treatment," Clinics in Perinatology, vol. 22, no. 2, pp. 457-479, 1995.

[11] C. A. Perez, J. M. Bustorff-Silva, E. Villasenor, E. W. Fonkalsrud, and J. B. Atkinson, "Surgical ligation of patent ductus arteriosus in very low birth weight infants: is it safe?" American Surgeon, vol. 64, no. 10, pp. 1007-1009, 1998.

[12] M. Narayanan, B. Cooper, H. Weiss, and R. I. Clyman, "Prophylactic indomethacin: factors determining permanent ductus arteriosus closure," Journal of Pediatrics, vol. 136, no. 3, pp. 330-337, 2000.

[13] S. Noori, M. McCoy, P. Friedlich et al., "Failure of ductus arteriosus closure is associated with increased mortality in preterm infants," Pediatrics, vol. 123, no. 1, pp. e138-e144, 2009.

[14] B. Van Overmeire, K. Smets, D. Lecoutere et al., "A comparison of ibuprofen and indomethacin for closure of patent ductus arteriosus," New England Journal of Medicine, vol. 343, no. 10, pp. 674-681, 2000.

[15] B. Van Overmeire, H. Van De Broek, P. Van Laer, J. Weyler, and P. Vanhaesebrouck, "Early versus late indomethacin treatment for patent ductus arteriosus in premature infants with 
respiratory distress syndrome," Journal of Pediatrics, vol. 138, no. 2, pp. 205-211, 2001.

[16] K. Itabashi, T. Ohno, and H. Nishida, "Indomethacin responsiveness of patent ductus arteriosus and renal abnormalities in preterm infants treated with indomethacin," Journal of Pediatrics, vol. 143, no. 2, pp. 203-207, 2003.

[17] J. Madan, J. Fiascone, V. Balasubramanian, J. Griffith, and J. I. Hagadorn, "Predictors of ductal closure and intestinal complications in very low birth weight infants treated with indomethacin," Neonatology, vol. 94, no. 1, pp. 45-51, 2008.

[18] N. Chorne, P. Jegatheesan, E. Lin, R. Shi, and R. I. Clyman, "Risk factors for persistent ductus arteriosus patency during indomethacin treatment," Journal of Pediatrics, vol. 151, no. 6, pp. 629-634, 2007.

[19] R. I. Clyman, "Mechanisms regulating the ductus arteriosus," Biology of the Neonate, vol. 89, no. 4, pp. 330-335, 2006.

[20] C. Pees, E. Walch, M. Obladen, and P. Koehne, "Echocardiography predicts closure of patent ductus arteriosus in response to ibuprofen in infants less than 28week gestational age," Early Human Development, vol. 86, no. 8, pp. 503-508, 2010.

[21] R. Greger and U. Windhorst, Comprehensive Human Physiology : From Cellular Mechanisms to Integration, Springer, New York, NY, USA, 1996.

[22] K. J. Crossley, B. J. Allison, G. R. Polglase, C. J. Morley, P. G. Davis, and S. B. Hooper, "Dynamic changes in the direction of blood flow through the ductus arteriosus at birth," Journal of Physiology, vol. 587, no. 19, pp. 4695-4704, 2009.

[23] R. I. Clyman, F. Mauray, M. A. Heymann, and C. Roman, "Influence of increased pulmonary vascular pressures on the closure of the ductus arteriosus in newborn lambs," Pediatric Research, vol. 25, no. 2, pp. 136-142, 1989.

[24] M. A. Heymann and A. M. Rudolph, "Control of the ductus arteriosus," Physiological Reviews, vol. 55, no. 1, pp. 62-78, 1975.

[25] R. I. Clyman, F. Mauray, and C. Roman, "Effect of gestational age on ductus arteriosus response to circulating prostaglandin E2," Journal of Pediatrics, vol. 102, no. 6, pp. 907-911, 1983. 


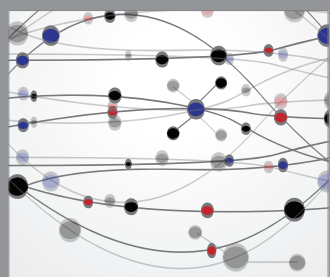

The Scientific World Journal
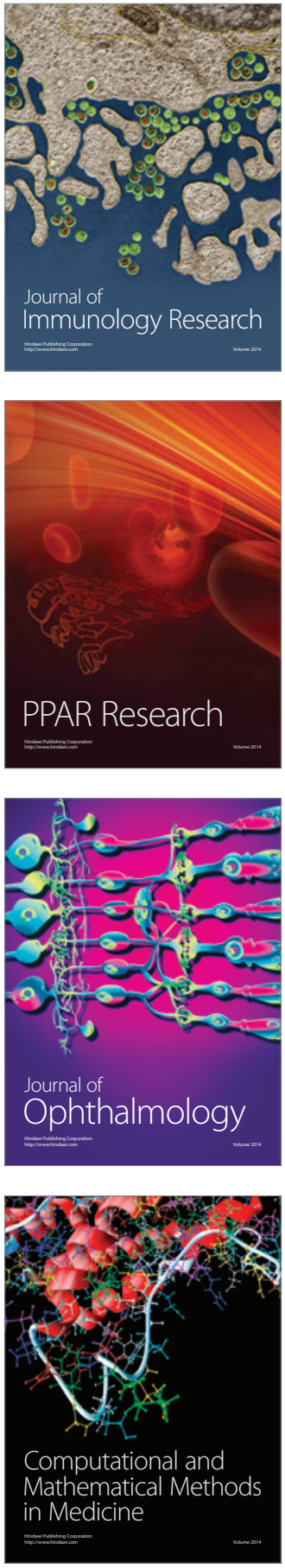

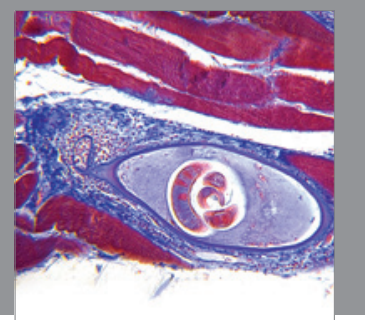

Gastroenterology

Research and Practice
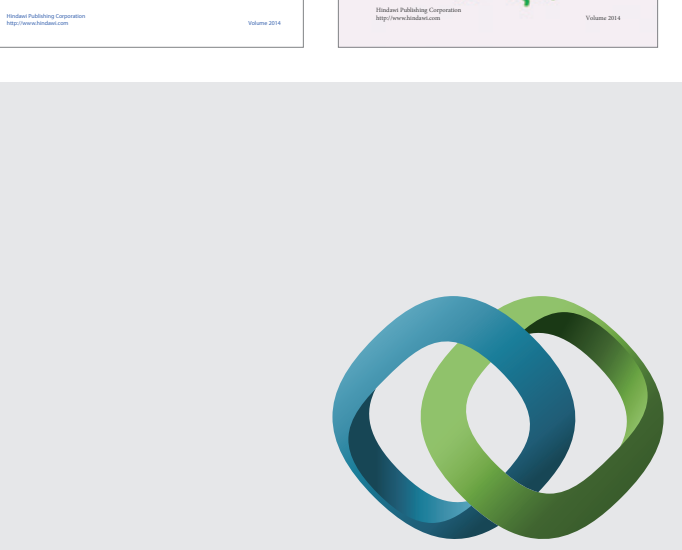

\section{Hindawi}

Submit your manuscripts at

http://www.hindawi.com
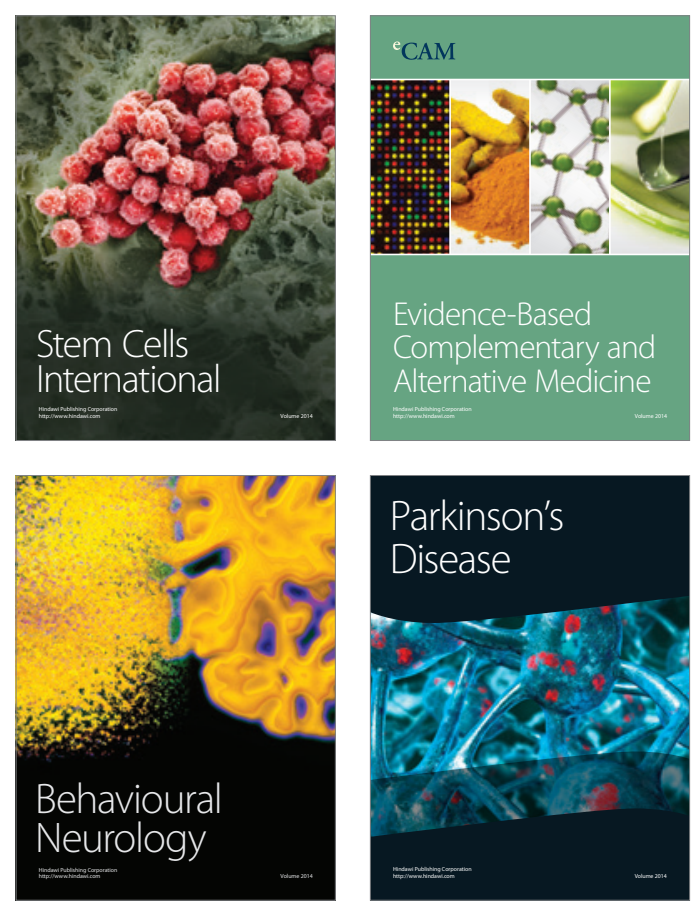

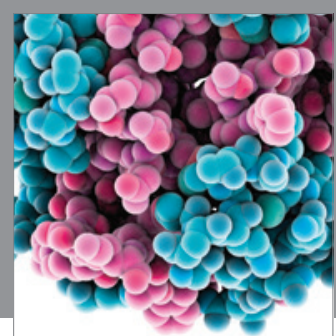

Journal of
Diabetes Research

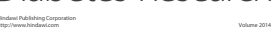

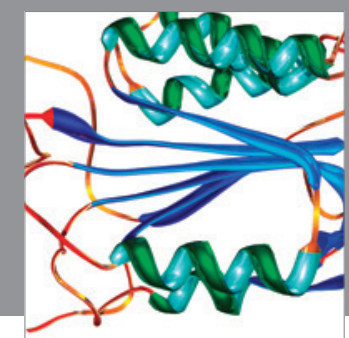

Disease Markers
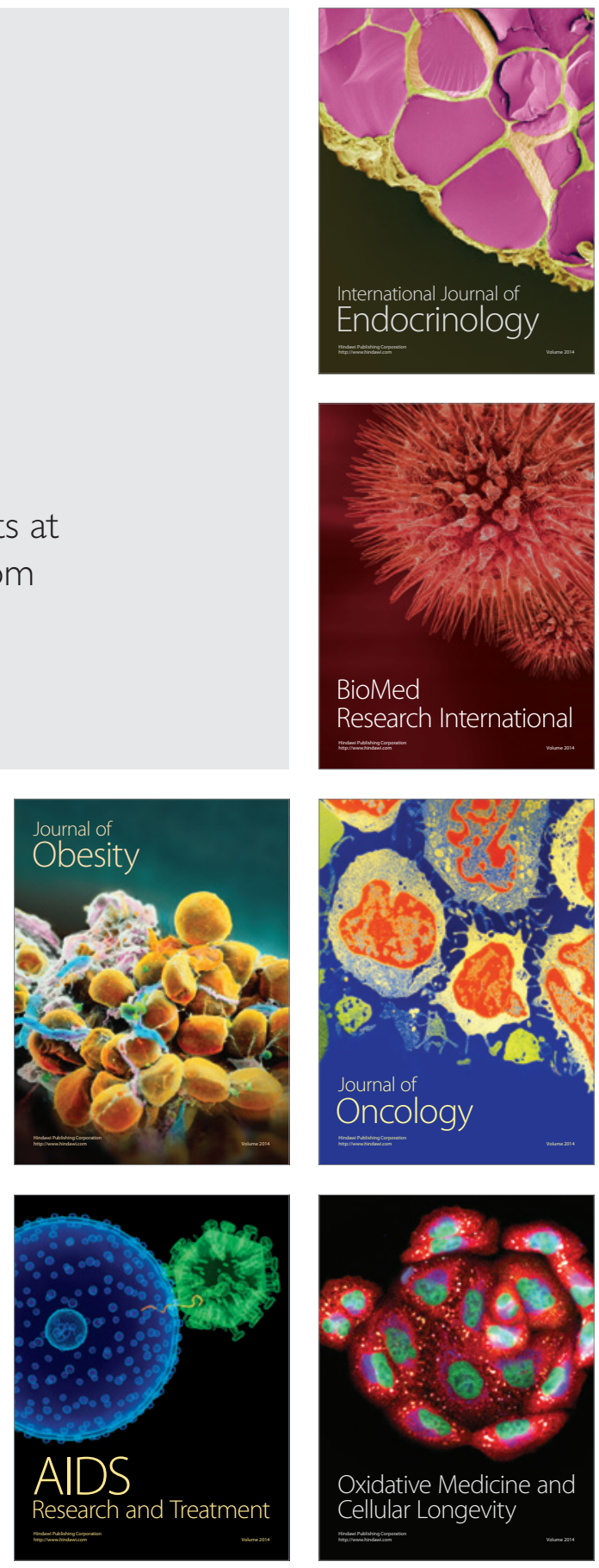